\title{
Acidez de la corteza de algunas especies nativas chilenas
}

\author{
Bark acidity of some Chilean native species
}

HERNAN POBLETE 1 , EDMONE ROFFAEL ${ }^{2}$

${ }^{1}$ Inst. de Tecnología de Productos Forestales, Universidad Austral de Chile, Casilla 567, Valdivia, Chile. E-mail: hpoblete@uach.cl

${ }^{2}$ Inst. für Holzbiologie und Holztechnologie. Universität Göttingen, Alemania.

\section{SUMMARY}

The amount of extracts and bark acidity from four different Chilean hardwoods (arrayán, coigüe, canelo and tepa) was measured and results revealed that the $\mathrm{pH}$ of the bark differed depending on the species. This is also valid for the buffering capacity of the extracts, the amount of volatile acids and the Stiasny value. The influence of a thermal treatment differed depending on the species.

Key words: bark, pH, volatile acids, buffer capacity, Stiasny.

\section{RESUMEN}

Se determinó la cantidad de extraíbles y la acidez en corteza de cuatro especies nativas chilenas (arrayán, coigüe canelo, tepa). Los resultados muestran que el valor de $\mathrm{pH}$ varía en forma importante dependiendo de la especie. Diferencias similares se registraron para la capacidad tampón alcalina, el contenido de ácidos volátiles y el número de Stiasny. También se pudo verificar que el efecto de un tratamiento térmico es variable dependiendo de la especie.

Palabras clave: corteza, pH, ácidos volátiles, capacidad tampón, Stiasny.

\section{INTRODUCCION}

La corteza se diferencia de la madera tanto por su morfología y, de forma muy importante por su composición química. El estudio sobre la acidez de la madera y de los ácidos que esta contiene han sido tratados por numerosos autores (1, 2). Sin embargo, son pocas las publicaciones que tratan la acidez de la corteza de los árboles (3).

La acidez de la madera, el contenido en ácidos libres y la capacidad tampón cambian con el tratamiento térmico dependiendo de la temperatura aplicada y de la duración del tratamiento $(2,4)$. Trabajos específicos sobre el efecto de los tratamientos térmicos sobre la acidez de la corteza se encuentran sólo esporádicamente (5). Los efectos de la acidificación de la madera y de la corteza son variados y dependen de la tecnología que se aplique.

Considerando la falta de información respecto al tema y la importancia que la acidez tiene en diferentes procesos industriales, en el presente trabajo se planteó determinar la acidez en muestras de corteza de diferentes especies latifoliadas nativas chilenas. Junto a las propiedades definidas anteriormente, se determinó la cantidad de polifenoles activos (número de Stiasny) que contienen las cortezas. Este tipo de compuestos es importante para evaluar la capacidad de las cortezas para producir taninos. 
BOSQUE 25(3): 73-78, 2004

Acidez de la corteza de algunas especies nativas chilenas

\section{MATERIAL Y METODOS}

Como material de estudio se seleccionaron cortezas de cuatro especies nativas chilenas. Las especies estudiadas fueron arrayán (Luma apiculata), canelo (Drimis winteri), coigüe (Nothofagus dombeyi), y tepa (Laurelia philippiana). Las especies coigüe y tepa fueron seleccionadas considerando la importancia de su participación en los bosques nativos chilenos. Ambas especies son además importantes en la fabricación de tableros donde la acidez es un factor a considerar. En el caso de canelo, se escogió esta especie por la rapidez de su crecimiento y la importancia potencial de la especie en la fabricación de tableros de partículas. La especie arrayán fue seleccionada por las características especiales de su corteza, de intenso color rojizo y normalmente libre del ataque de plantas parásitas.

Para efectuar las determinaciones se extrajeron de trozas recién cortadas, porciones de corteza a la altura de pecho (DAP). Los árboles seleccionados para este efecto se encontraban en predios de la décima Región de Los Lagos, precordillera andina.

La corteza recolectada se trituró y se determinó el contenido de extraíbles, el valor de $\mathrm{pH}$ de los extractos, la cantidad de ácidos y la reactividad de los extractos frente al formaldehido según el método de Stiasny.

Determinación de los extraíbles. Las muestras de corteza fueron extraídas de acuerdo con los siguientes métodos:

-Etanol-Tolueno
- $\mathrm{NaOH}(1 \%)$
-Agua

según TAPPI T204 según TAPPI T212 según TAPPI T207

La cantidad de extraíbles se expresó como porcentaje del peso seco de la muestra.

Determinación del valor de $p H$. La determinación del valor de $\mathrm{pH}$ se efectuó en los extractos en agua. Para ello se tomaron $10 \mathrm{~g}$ de corteza, se mezclaron con $150 \mathrm{ml}$ de agua destilada y se agitaron por 24 horas. Finalmente se determinó el valor de $\mathrm{pH}$ por medio de un pH-metro en los extractos.
Determinación del número de Stiasny. Con este método se determina la reactividad de los extraíbles polifenólicos en presencia de formaldehido. Para la determinación del número de Stiasny se tomaron $50 \mathrm{ml} \mathrm{del} \mathrm{extracto} \mathrm{obtenido}$ para la determinación del valor de $\mathrm{pH}$. Esta solución se mezcló con $5 \mathrm{ml} \mathrm{HCl}$ (concentrado) y 10 $\mathrm{ml}$ de solución de formaldehido (concentrado). La mezcla se mantuvo en ebullición bajo reflujo por $30 \mathrm{~min}$. El precipitado obtenido se filtró con vacío en un filtro de vidrio G3 (previamente secado y tarado) y se lavó con agua destilada. Posteriormente se secó por dos horas a $120^{\circ} \mathrm{C}$ y se pesó después de enfriarlo en desecador. Paralelamente se pesó el remanente seco de corteza. El número de Stiasny se calculó como la cantidad de precipitado expresada como porcentaje del residuo seco de corteza:

$$
\text { Número de Stiasny }=\frac{\text { Precipitado }(\mathrm{g}) \text { x } 100}{\text { Remanente } \operatorname{seco}(\mathrm{g})}
$$

Tratamientos térmicos. Las muestras de corteza se trataron de acuerdo con la metodología de los frascos (WKI) para la determinación de la emanación de formaldehido en tableros. De esta forma se tomaron muestras de $2 \mathrm{~g}$ de corteza molida, se ubicaron en recipientes de aluminio y se colgaron en el interior de recipientes (frascos) de acero con un volumen aproximado de $600 \mathrm{~cm}^{3}$. En estos frascos se incluyeron $20 \mathrm{ml}$ de agua destilada, se cerraron herméticamente y se sometieron por 24 , 48, 72 y 144 horas en estufa a $50{ }^{\circ} \mathrm{C}$ y $103{ }^{\circ} \mathrm{C}$. Para cada tipo de corteza y tratamiento se efectuaron 6 repeticiones.

Luego del tratamiento térmico los recipientes se enfriaron en agua y se rescató de su interior la solución de agua con los extraíbles emanados. En estos extractos se efectuaron las determinaciones de valor de $\mathrm{pH}$, capacidad tampón y contenido de ácidos volátiles.

El valor de $\mathrm{pH}$ se midió por medio de $\mathrm{pH}$ metro digital. La cantidad de ácidos volátiles obtenidos de la corteza se determinó por cromatografía iónica. La capacidad tampón se determinó como el consumo de álcali para neutralizar los extractos $(\mathrm{pH}=7)$. Esta propiedad se expresó como $\mathrm{mmol} / \mathrm{kg}$. 


\section{CUADRO 1}

Contenido de extraíbles en corteza de algunas especies nativas chilenas. Amount of extracts in the bark of some Chilean species.

\begin{tabular}{|l|r|c|c|c|}
\hline \multirow{2}{*}{$\begin{array}{l}\text { Solvente } \\
\text { empleado }\end{array}$} & \multicolumn{4}{|c|}{ Extraíbles por tipo de corteza (\% bms) } \\
\cline { 2 - 5 } & Coigüe & Tepa & Canelo & Arrayán \\
\hline Agua fría & 5,01 & 12,16 & 11,96 & 13,18 \\
Agua caliente & 11,45 & 15,58 & 16,39 & 24,00 \\
NaOH (1\%) & 30,13 & 37,90 & 36,88 & 50,43 \\
Etanol-Tolueno & 7,67 & 11,71 & 54,88 & 14,64 \\
\hline
\end{tabular}

bms: base madera seca

\section{RESULTADOS}

Extraíbles. Como se mencionara en la metodología, se emplearon como solventes etanol tolueno $(1: 1)$, agua fría y caliente $\left(100^{\circ} \mathrm{C}\right)$ e hidróxido de sodio diluido (1\%). Los resultados de los contenidos de extraíbles de las diferentes cortezas se presentan en el cuadro 1.

En el cuadro 1 se observa que con la extracción en agua fría no se obtienen cantidades de extracto que superen a un $15 \%$ del peso de la corteza. Con el aumento de la temperatura del agua la cantidad de sustancias que se diluyen aumenta significativamente. Con la solución de hidróxido de sodio al $1 \%$ la cantidad de material disuelto varía entre $30,1 \%$ y $50,4 \%$. La extracción con etanol-tolueno da por resultado cantidades de extraíbles más variables dependiendo de la especie. En este último caso los valores van de $7,7 \%$ a $54,9 \%$

Los resultados permiten concluir que el tipo de extraíbles obtenidos de las cortezas analizadas presentan diferencias importantes en su cantidad y naturaleza. Lo anterior lo demuestra el hecho de que la corteza de tepa registra la cantidad más alta de extraíbles en $\mathrm{NaOH}$, mientras que canelo lo hace con etanol-tolueno.

Valor de $\mathrm{pH}$ de los extraíbles en agua fría. El valor de $\mathrm{pH}$ de los extractos en agua fría se determinó luego de 24 horas de agitación mecánica. Los resultados de esta extracción se presentan en el cuadro 2.

Los valores de $\mathrm{pH}$ presentan diferencias importantes entre las especies. Así los extractos de la corteza de tepa son neutros $(\mathrm{pH}=7,2)$ y los de

\section{CUADRO 2}

Valor de $\mathrm{pH}$ y número de Stiasny de los extractos en agua fría.

$\mathrm{pH}$ value and Stiasny number of cold water extracts.

\begin{tabular}{|l|c|c|c|c|}
\hline \multirow{2}{*}{ Propiedad } & \multicolumn{4}{|c|}{ Tipo de corteza } \\
\cline { 2 - 5 } & Coigüe & Tepa & Canelo & Arrayán \\
\hline Valor de $\mathrm{pH}$ & 4,7 & 7,2 & 6,1 & 3,9 \\
Stiasny $\left(\mathrm{N}^{\circ}\right)$ & 78,89 & 18,67 & 10,38 & 28,88 \\
\hline
\end{tabular}

arrayán muy ácidos $(\mathrm{pH}=3,9)$. Las cortezas de coigüe y canelo tienen valores intermedios con 4,7 y 6,1, respectivamente.

Los valores obtenidos son similares a los reportados para madera de las mismas especies. Albin (6) registra un $\mathrm{pH}$ de 4,3 en coigüe, 6,2 para tepa y 5,8 en canelo. Para la madera o corteza de arrayán no se encontraron antecedentes.

Número de Stiasny de los extractos. A los extraíbles de la corteza, obtenidos luego de 24 horas de agitación en agua fría, se les determinó su capacidad de reaccionar frente al formaldehido. Esta determinación da como resultado el denominado número de Stiasny. La determinación de este valor es importante por cuanto indica qué porcentaje de los extraíbles obtenidos tienen la capacidad de reaccionar con el formaldehido, lo que indirectamente se relaciona con la posibilidad de obtener taninos para uso industrial. Los resultados de esta determinación se presentan en el cuadro 2 . 
BOSQUE 25(3): 73-78, 2004

Acidez de la corteza de algunas especies nativas chilenas

Los valores más altos se obtuvieron con corteza de coigüe $(78,89)$ y con arrayán $(28,88)$. Tepa y canelo registran los más bajos, con 18,67 y 10,38 , respectivamente.

Los extractos de las cortezas muestran grandes diferencias en su contenido de compuestos polifenólicos con capacidad de reacción. Los extractos de coigüe son más ricos en grupos polifenólicos activos en comparación con los de canelo. Comparativamente, la reactividad de los extractos de coigüe son similares a los de Picea abies en Europa, mientras que en canelo y tepa la reactividad es más parecida a la de los extractos de Pinus sylvestris (7).

Determinación del valor de $\mathrm{pH}$, capacidad tampón y ácidos volátiles luego de un tratamiento térmico. Los resultados de las determinaciones de valor de $\mathrm{pH}$, capacidad tampón y de la cantidad emanada de ácidos acético y fórmico, luego de un tratamiento térmico, se presentan en el cuadro 3.

Los valores de $\mathrm{pH}$ disminuyen en forma drástica al aumentar la temperatura del tratamiento.

El efecto de la duración del tratamiento con $50{ }^{\circ} \mathrm{C}$ sobre el valor de $\mathrm{pH}$ es claro en todas las especies salvo en coigüe. En la corteza de arrayán, canelo y tepa se produce una acidificación al prolongar el tratamiento de 24 a 144 horas con $50{ }^{\circ} \mathrm{C}$. En coiguie esta acidificación no se observa. Con $50{ }^{\circ} \mathrm{C}$ la corteza de arrayán reacciona entregando la mayor cantidad de ácidos y esta cantidad se incrementa notablemente al prolongarse el tratamiento.

El cambio de tratamiento de $50{ }^{\circ} \mathrm{C}$ a $103{ }^{\circ} \mathrm{C}$ provoca un cambio significativo de la acidez. La duración del tratamiento a $103{ }^{\circ} \mathrm{C}$ provoca una acidificación del material, salvo en el caso de tepa.

La corteza de la especie tepa es la que sufre un cambio más marcado al aumentar la temperatura. El resto de las cortezas muestran una acidificación del material pero en menor escala (figura 1).

Al comparar los valores de $\mathrm{pH}$ obtenidos con las dos temperaturas (figura 1) se observa que el grado de acidificación es diferente dependiendo de la especie de corteza.

Los valores muy bajos y constantes impidieron determinar adecuadamente la capacidad tampón en los tratamientos a $50^{\circ} \mathrm{C}$. Los resultados de la capacidad tampón demostraron que no existe efecto alguno al tratar las cortezas con $50{ }^{\circ} \mathrm{C}$.

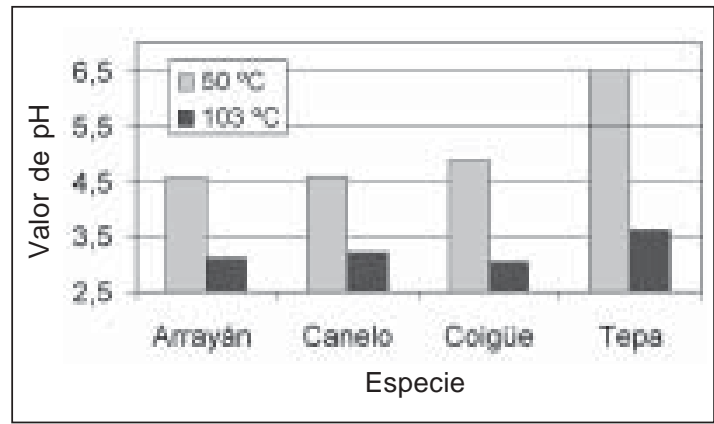

Figura 1. Promedio del valor de $\mathrm{pH}$ en extractos obtenidos luego de un tratamiento con $50^{\circ} \mathrm{C}$ y $103^{\circ} \mathrm{C}$

Average $\mathrm{pH}$ value of extracts obtained after treatment at $50^{\circ} \mathrm{C}$ and $103^{\circ} \mathrm{C}$

$\mathrm{Al}$ elevar la temperatura a $103{ }^{\circ} \mathrm{C}$ se registra una clara tendencia (cuadro 3) a aumentar la capacidad tampón a medida que aumenta la duración del tratamiento. Este comportamiento es especialmente marcado en el caso de coigüe.

En términos generales se pudo determinar que la magnitud del aumento de la capacidad tampón depende de la especie de corteza.

Con un tratamiento a $50{ }^{\circ} \mathrm{C}$ (figura 2), la corteza de la especie arrayán luego de 144 horas es la que entrega la mayor cantidad de ácidos. En todas las especies estudiadas se pudo observar un aumento de la cantidad de ácidos emanados al incrementarse el tiempo de tratamiento.

Las magnitudes de las emanaciones a $50{ }^{\circ} \mathrm{C}$ son muy pequeñas si se comparan con las cantidades emanadas con $103{ }^{\circ} \mathrm{C}$. En la figura 3 se

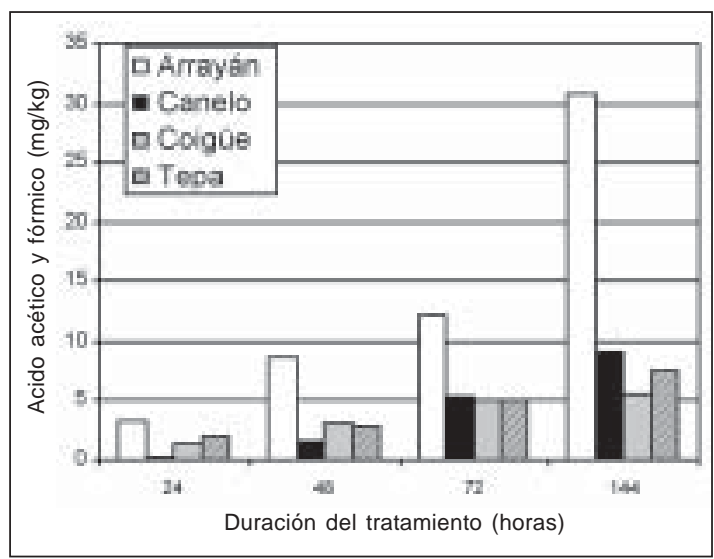

Figura 2. Cantidad de ácido acético y fórmico (suma de ambos) luego de tratamientos a $50{ }^{\circ} \mathrm{C}$ por diferentes períodos de tiempo.

Amount of acetic and formic acid (sum of both) after treatment at $50^{\circ} \mathrm{C}$ for different time periods. 
BOSQUE 25(3): 73-78, 2004 Acidez de la corteza de algunas especies nativas chilenas

\section{CUADRO 3}

Valor de $\mathrm{pH}$, capacidad tampón y ácidos volátiles en corteza de algunas especies nativas chilenas tratadas con $50{ }^{\circ} \mathrm{C}$ y $103{ }^{\circ} \mathrm{C}$ por 24 a 144 horas.

$\mathrm{pH}$ value, buffer capacity and volatile acids in bark of some Chilean species treated at $50{ }^{\circ} \mathrm{C}$ and $103{ }^{\circ} \mathrm{C}$ for 24 to 144 hours.

\begin{tabular}{|c|c|c|c|c|c|c|}
\hline \multirow[b]{2}{*}{ Especie } & \multicolumn{2}{|c|}{ Tratamiento } & \multirow[b]{2}{*}{ Valor $\mathrm{pH}$} & \multirow[b]{2}{*}{$\begin{array}{l}\text { Capacidad Tampón } \\
(\mathrm{mmol} / \mathrm{kg})\end{array}$} & \multicolumn{2}{|c|}{ Acidos volátiles } \\
\hline & $\begin{array}{l}\text { Temp. } \\
\left({ }^{\circ} \mathrm{C}\right)\end{array}$ & $\begin{array}{l}\text { Tiempo } \\
\text { (Horas) }\end{array}$ & & & $\begin{array}{l}\text { Acético } \\
(\mathrm{mg} / \mathrm{kg})\end{array}$ & $\begin{array}{l}\text { Fórmico } \\
(\mathrm{mg} / \mathrm{kg})\end{array}$ \\
\hline \multirow{8}{*}{ Arrayán } & 50 & 24 & 5,1 & - & 3,3 & 0,0 \\
\hline & 50 & 48 & 4,6 & - & 8,7 & 0,0 \\
\hline & 50 & 72 & 4,3 & - & 11,9 & 0,3 \\
\hline & 50 & 144 & 4,2 & - & 29,2 & 1,6 \\
\hline & 103 & 24 & 3,3 & 59,5 & 355,8 & 64,7 \\
\hline & 103 & 48 & 3,3 & 108,9 & 676,3 & 185,4 \\
\hline & 103 & 72 & 2,9 & 125,9 & 723,1 & 151,0 \\
\hline & 103 & 144 & 2,9 & 149,4 & 879,6 & 157,5 \\
\hline \multirow{8}{*}{ Canelo } & 50 & 24 & 5,1 & - & 0,0 & 0,3 \\
\hline & 50 & 48 & 4,7 & - & 1,2 & 0,3 \\
\hline & 50 & 72 & 4,5 & - & 4,0 & 1,3 \\
\hline & 50 & 144 & 4,0 & - & 6,0 & 3,0 \\
\hline & 103 & 24 & 3,3 & 29,6 & 204,2 & 95,8 \\
\hline & 103 & 48 & 3,2 & 92,5 & 312,0 & 122,5 \\
\hline & 103 & 72 & 3,2 & 109,9 & 376,7 & 143,0 \\
\hline & 103 & 144 & 3,1 & 140,3 & 712,3 & 228,0 \\
\hline \multirow{8}{*}{ Coigüe } & 50 & 24 & 5,0 & - & 1,4 & 0,0 \\
\hline & 50 & 48 & 4,7 & - & 3,2 & 0,0 \\
\hline & 50 & 72 & 4,8 & - & 4,8 & 0,4 \\
\hline & 50 & 144 & 5,0 & - & 4,4 & 1,2 \\
\hline & 103 & 24 & 3,3 & 35,4 & 243,8 & 111,9 \\
\hline & 103 & 48 & 3,0 & 84,2 & 534,1 & 213,5 \\
\hline & 103 & 72 & 3,0 & 140,8 & 855,8 & 410,7 \\
\hline & 103 & 144 & 2,8 & 236,0 & 976,0 & 382,7 \\
\hline \multirow{8}{*}{ Tepa } & 50 & 24 & 6,8 & - & 1,3 & 0,7 \\
\hline & 50 & 48 & 6,6 & - & 2,8 & 0,0 \\
\hline & 50 & 72 & 6,4 & - & 3,6 & 1,6 \\
\hline & 50 & 144 & 6,2 & - & 6,6 & 0,9 \\
\hline & 103 & 24 & 3,6 & 14,9 & 114,5 & 71,5 \\
\hline & 103 & 48 & 3,3 & 40,0 & 270,1 & 213,6 \\
\hline & 103 & 72 & 3,8 & 46,0 & 295,2 & 186,7 \\
\hline & 103 & 144 & 3,8 & 91,9 & $1.083,5$ & 891,7 \\
\hline
\end{tabular}


BOSQUE 25(3): 73-78, 2004

Acidez de la corteza de algunas especies nativas chilenas

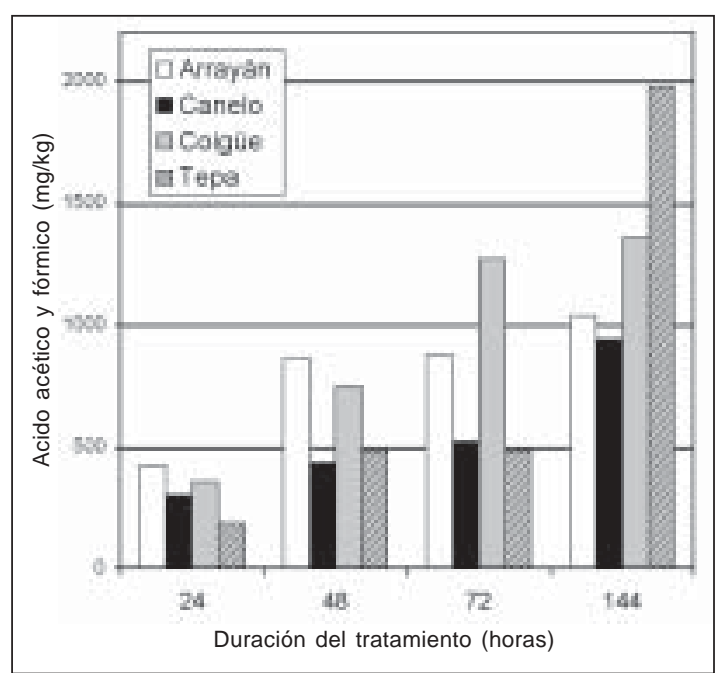

Figura 3. Cantidad de ácido acético y fórmico (suma de ambos) luego de tratamientos a $103{ }^{\circ} \mathrm{C}$ por diferentes períodos de tiempo.

Amount of acetic and formic acid (sum of both) after treatment at $103^{\circ} \mathrm{C}$ for different time periods.

observa que el nivel de emanaciones es casi 100 veces mayor que lo registrado con $50^{\circ} \mathrm{C}$. Tal como en el tratamiento inicial, $50{ }^{\circ} \mathrm{C}$, se midió un aumento de la cantidad de ácidos al aumentar el tiempo de tratamiento. La cantidad de ácidos emanados por tepa es la mayor, habiéndose detectado corrosión en los materiales usados con esta corteza.

\section{CONCLUSIONES}

Existen diferencias notorias en la acidez de las cortezas estudiadas. El tipo de corteza, la temperatura de tratamiento y el tiempo de tratamiento tienen un efecto importante sobre las característi- cas químicas estudiadas. El tipo de corteza y la temperatura aparecen como las variables más importantes. La especie tepa presentó los valores de pH más alcalinos. Esta especie libera una cantidad moderada de ácidos, pero no cuando se aplica el tratamiento más severo $\left(103{ }^{\circ} \mathrm{C}\right.$ por 144 horas $)$.

Los contenidos de compuestos polifenólicos activos son también muy variables entre las especies, siendo la corteza de coigüe la que presenta el contenido más alto de estos compuestos.

Los resultados obtenidos y la necesidad de conocer mejor la corteza como materia prima permiten concluir que en el futuro es necesario hacer estudios más acabados sobre sus características tecnológicas.

\section{BIBLIOGRAFIA}

(1) Poblete, H., E. ROFFAEL. Über chemische Veränderungen in Holzspänen bei der Herstellung von Harnstoff-Formaldehydharz gebundenen Spanplatten. Holz als Roh- und Werkstoff, 1985, vol. 43, p. 57-62.

(2) ROFFAEL, E., H. POBLETE., M. TORRES. Über die Acidität von Kern- und Splintholz der Kastanie (Castanea sativa) aus Chile. Holz als Roh- und Werkstoff, 2000. vol. $58, \mathrm{~N}^{\circ} 1-2$, p. $120-122$

(3) PRASETYA B . E ROFFAEL Zur Acidität der Rinde einiger Nadelbaumarten. Stand der Kenntnisse und eigene Untersuchungen. Holz als Roh- und Werkstoff, 1990, vol 48, p. 429-435.

(4) CHOON, K., E. ROFFAEL. The Acidity of five Hardwood Species. Holzforschung, 1990, vol. 44, p. 53-58.

(5) PRASETYA, B. Beiträge zum Verhalten extraktstoffreicher Rinden, am Beispiel der Fichtenrinde, in chemisch-technologischen Prozessen. Dissertation. Forstlichen Fakultät der Georg August Universität zu Göttingen. 1992, p. 260.

(6) ALBIN, R. Determinación del pH en diversas especies de los renovales de la provincia de Valdivia. Bosque (Chile) 1975 , vol. $1, \mathrm{~N}^{\mathrm{o}} 1$, p. 3-5

(7) ROFFAEL, E. Über die Reaktivität von wäbrigen Rindenextrakten gegenüber Formaldehyd. Adhäsion, 1976 , vol. $20, \mathrm{~N}^{\circ}$. 11 , p. $306-311$. 